\title{
La recomposición del sistema de protección social
}

Reflexiones en relación a la intervención social del Estado en el contexto pos neoliberal

Juan Bautista Sala Juan Bautista Sala Licenciado en Trabajo Social (UNLP) Especialista en políticas sociales (UNLP) Docente de la Facultad de Trabajo Social (UNLP)

E-mail: bautistasala@gmail.com 
Resumen

El presente trabajo se desarrolla en relación a la afirmación que, desde el inicio del nuevo siglo, la Argentina, junto con su recuperación económica ha emprendido un camino de recuperación de instituciones sociales. No sólo es una reconstrucción y actualización de viejas conquistas, también se ha avanzado en la creación de planes y programas sociales, como la Asignación Universal por Hijo, que dan un salto conceptual y, por lo tanto, interpelan al Estado, a las instituciones políticas y a las ciencias sociales. En tal sentido, el objetivo del presente trabajo es dar cuenta del cambio de enfoque en del Sistema de Protección Social Argentino, caracterizando su situación actual e identificando las rupturas y continuidades. A partir de reconocer los modelos típicos construidos históricamente se profundizará en el análisis del caso argentino identificando las particularidades que adopta en cada uno de los modelos de desarrollo trabajados. Se entiende protección social como un concepto abarcativo que incluye toda la intervención social del Estado: mecanismos e instituciones destinadas a la protección social de los ciudadanos. Se incluye aquí la asistencia social, la seguridad social y los servicios sociales en general, como pertenecientes a un sistema construido históricamente con una coagulación coyuntural, el cual va variando en su función y composición con el devenir político de los países, sus necesidades, sus recursos y capacidades.

\section{Palabras claves}

Protección Social - Políticas públicas - Estado

\section{Abstract}

This paper takes place in relation to the claim that since the beginning of the new century, Argentina, together with its economic recovery has embarked on a path of recovery social institutions. It is not just a reconstruction and renovation of old conquests, also it has been made in creating plans and social programs such as universal child allowance, which give a conceptual leap and, therefore, question the State, political institutions and social sciences. Following this direction, it will deepen the analysis of the Argentinian Social Protection System, in order to characterize the current situation and identify the ruptures and continuities. In this regard, the objective of this work is to account for the shift in focus in the argentinian social protection system, characterizing the current situation and identifying the ruptures and continuities. From recognizing historically typical models built will deepen the analysis of the Argentine case identifying the particularities adopted in each of the development models worked. Social protection is understood as a comprehensive concept that includes all the social intervention by the State: mechanisms and institutions for the social protection of citizens. It includes social care, social security and social services in general, as belonging to a historically built a temporary clotting system, which will vary in function and composition with the political future of the country, its needs, its here resources and capabilities.

\section{Kew Words}

Social Protection - Social Policies - State 


\section{Introducción}

El presente trabajo se inicia a partir de afirmar que, desde el inicio del nuevo siglo, la Argentina, junto con su recuperación económica ha emprendido un camino de recuperación de instituciones sociales. Pero no sólo es una reconstrucción y actualización de viejas conquistas, también se ha avanzado en la creación de planes y programas sociales que dan un salto conceptual, y por lo tanto interpelan al Estado a las instituciones políticas y a las ciencias sociales, principalmente la asignación universal por hijo pero también otros ejemplos como la moratoria previsional, las cuales se potencian a partir de lo que aquí se identifica como una segunda generación de políticas sociales del gobierno actual a partir de la recuperación de los fondos previsionales.

Siguiendo esta dirección, se profundizará en el análisis del sistema de protección social Argentino, con el fin de caracterizar la su situación actual e identificar las rupturas y continuidades.

En el primer apartado se identifican los modelos de protección social típicos construidos históricamente. Cabe aclarar que se entiende protección social como un concepto abarcativo que incluye todo la intervención social del Estado ${ }^{1}$. Mecanismos e instituciones destinadas a la protección social de los ciudadanos. Se incluye aquí la asistencia social, la seguridad social y los servicios sociales en general, como pertenecientes a un sistema construido históricamente con una coagulación coyuntural, el cual va variando en su función y composición con el devenir político de los países, sus necesidades, sus recursos y sus capacidades.

En el segundo apartado se identifican las formas que han tomado históricamente los sistemas de protección. Y, posteriormente, se profundiza en el análisis del caso argentino identificando las particularidades que adopta en cada uno de los modelos de desarrollo trabajados.

1 Golbert et al (2012). 


\section{Orígenes de la protección social}

El sistema de protección social moderno tiene sus orígenes más acabados en la Alemania de fines de siglo XIX con la creación del Seguro Social por parte del primer ministro de Prusia Otto Von Bismark. Esta iniciativa se llevó adelante en pleno proceso industrializador donde la explotación y las malas condiciones de trabajo eran el centro de la cuestión social.

Ante las presiones, principalmente, de la clase trabajadora y contra el avance del socialismo, hacia 1883 se crea el seguro contra enfermedad, en 1884 el seguro contra accidentes de trabajo, en 1889 por invalidez y vejez. En 1911 se crea el seguro de supervivencia, y el código de seguros sociales. De esta manera se consigura el seguro social, una de las principales instituciones del Estado de Bienestar que rápidamente se desarrollaría en Europa occidental y que posteriormente tendría su versión latinoamericana.

El seguro social está inspirado en los seguros privados y se basa en los principios de solidaridad que surgieron con la formación de la clase trabajadora industrial, no obstante, mantiene un espíritu meritocrático en tanto que los individuos recibirán prestaciones acordes a los aportes: "El esquema financiero del modelo del seguro social revela una asociación entre política social e inserción laboral" (Fleury et al, 2000:8); éste depende de la contribución del trabajador, el empleador y el Estado; no obstante se establece una relación directa entre la contribución y la prestación por lo que tiende a reproducir la segmentación propia del mercado laboral.

Más de medio siglo después, en plena segunda guerra mundial y en un contexto de alto desempleo, William Beveridge presenta el "Informe al Parlamento acerca de la seguridad social y de las prestaciones que de ella se derivan" que había sido requerido por el Ministro de Trabajo Inglés. El Informe, conocido como Plan Beveridge, de 1942, proponía garantizar un mínimo de subsistencia que abarcara la más amplia gama de situaciones de necesidades posibles y se basaba en tres pilares: salud universal, empleo seguro y asignación por hijo (Roca et al, 2012:29). En su concepción se entendía que "el bien- 
estar de la sociedad no es un efecto de la relación laboral, sino una obligación del estado hacia sus ciudadanos" (Beveridge, 1909 apud Roca et al, 2012:29).

En este caso, el carácter igualitario del modelo está basado en el aseguramiento de un patrón mínimo de beneficios de forma universalizada, independientemente de las contribuciones anteriores. El acceso a los beneficios sociales depende únicamente de la necesidad de los individuos (Fleury et al, 2000:8). El carácter institucional de este modelo se da, en tanto que la administración como el financiamiento del sistema, están regulados y gestionados por el Estado a través del reparto solidario de los recursos.

Así, la seguridad social, inspirada en principios universales de solidaridad, equidad y justicia social, rescata la responsabilidad estatal y de la sociedad en la protección social y se erige como derecho fundamental (Mejía, 2007:3).

Estas formas de intervención (el seguro social y la seguridad social) se suman al clásico modelo de asistencia social propio del período liberal de transición capitalista, ya sea con carácter caritativo o filantrópico. Propio de los períodos liberales la exaltación del lugar del mercado en la regulación social brinda al Estado y a la acción pública un lugar residual y/o complementario destinado a los grupos sociales que no pueden insertarse en la dinámica del desarrollo de la sociedad.

Así, se configuran los tres principales modelos de protección social: el Modelo del Seguro Social, el de Seguridad Social y el de Asistencia Social que, de diversas maneras, van a ser instituciones fundantes de los Estados de Bienestar que terminaron de moldearse entre la gran crisis de los años 30 y la Segunda Guerra Mundial; y se desarrollaron hasta la crisis del petróleo a mediados de los 70 .

Si bien parecen descriptas en un sentido evolutivo, las diferentes formas de protección social responden a los modelos de Estado predominantes en cada período histórico y de una u otra manera las diferentes formas de intervención coexisten en la actualidad tomando diferentes particularidades según sea el proyecto político coyuntural. 


\section{El sistema de protección social en Argentina. Protección social y modelo de desarrollo}

En América Latina, los sistemas de protección social se moldearon con las particularidades de los diversos regímenes políticos y económicos que caracterizan la región. Mesa Lagos (1985) clasifica a los países de acuerdo al momento en que incorporan instituciones de la protección social en pioneros, intermedios y tardíos. Los pioneros (Argentina, Brasil, Uruguay y Chile) quienes tuvieron los primeros desarrollos entre el 1920 y el 1930, intermedios aquellos que incursionaron entre 1940 y 1950 (México. C. Rica, Panamá, Países Andinos) y, por último, los tardíos (países de Centroamérica y El Caribe).

A partir de los modelos de bienestar que propone Esping-Andersen (1993) (liberal, corporativo y socialdemócrata) existe un consenso en caracterizar al sistema de seguridad Argentino como "mixto". Es decir, predominó un modelo de tipo corporativo en los regímenes de seguridad social y uno de orientación universal en el campo de la educación y en los principios de la salud pública. Sin embargo, este último sector sufrió una temprana sangría de recursos e ineficiente gestión, provocando que a partir de los 60 las políticas se focalicen sólo en la población indigente, constituyendo un modelo de tipo residual. Más allá de estas diferenciaciones, durante el Estado Social prevaleció un modelo de ciudadanía estratificado o regulado, articulado en torno a los sectores integrados al mercado de trabajo formal. Bajo este marco, los trabajadores se constituían como categoría social con derechos específicos y el trabajo asalariado aparecía asociado a una red de protecciones sociales con base jurídica. Cortés y Marshall (1994) analizan esta intrínseca relación entre política social y mercado de trabajo, definiendo que las políticas sociales adaptan la estructura del mercado de trabajo a las necesidades de la estrategia de acumulación ${ }^{2}$.

2 Una de las hipótesis de dicho artículo es que la orientación de la política social no puede ser incompatible con el modelo de crecimiento, es decir que la pauta de la intervención social del Estado varía de acuerdo con las condiciones que establece el modelo de crecimiento económico (Cortes y Marshall, 1994). 
Desde el primer período, la intervención social del Estado se había construido y consolidado como un sistema de protección del trabajo que respondía en cierta medida a las exigencias de las organizaciones obreras. Durante 1943/1976 la política social acompañó e impulsó la estrategia económica de sustitución de importaciones. En efecto, durante esta etapa la regulación estatal modificó profundamente las condiciones de trabajo y el sistema de relaciones laborales. Se instituyen las vacaciones pagas, aguinaldo, indemnización por despido, etc., así como también se desarrolla la seguridad social, que establece que los empleadores debían proveer servicios sociales básicos como salud y medicamentos, comida, vestimenta. Por otra parte, la política salarial, los subsidios indirectos al consumo de alimentos y el control de alquileres, son ejemplos del incremento de la intervención social del Estado durante este período que, mediante el fortalecimiento del poder adquisitivo, buscaban impulsar el desarrollo del mercado interno. En este marco el Estado cumplía un rol importante en el entrenamiento y preservación de la fuerza de trabajo.

Durante esta etapa se expandió y consolidó un modelo de intervención social protectora que tenía tres ejes: un soporte político caracterizado por un pacto corporativo entre los sindicatos, el Estado y los empleadores. Se establecía que a cambio de la moderación sindical se proveería a los trabajadores servicios universales y continuidad en el trabajo. Un soporte social que tenía como eje una relación asalariada que establecía el conjunto de derechos y obligaciones de asalariados y empleadores en el ámbito del lugar de trabajo (vacaciones, aguinaldo, etc.) y de la protección social (seguro de desempleo y enfermedad). Un modelo de familia basado en una implícita división del trabajo donde el varón aparecía como el único proveedor de ingresos, mientras la mujer se ocupaba de las tareas reproductivas.

En resumen, las políticas sociales que se consolidaron a partir de los años 40, se construyeron sobre los principios del seguro social bismarckiano, con derechos y obligaciones normados sobre la categoría de "trabajador" (particularmente asalariado) y no sobre la de "ciudadano". En este marco, la ausencia del seguro de desempleo se comprende tanto por la idea de que la economía funcionaba a niveles 
cercanos al pleno empleo, como por la propia lógica del modelo corporativo: los derechos se legitimaban, y eran legitimados, a través de la pertenencia a una corporación. Asimismo, el proceso de difusión de los beneficios de las políticas sociales se basaba en el sentido de la expansión vertical, resultando de un efecto "cascada" desde los grupos mejor ubicados hacia el resto. Mientras, las políticas asistenciales dirigidas a los grupos no integrados al mercado laboral, aparecen como un elemento residual en esta dinámica (Barbeito y Lo Vuolo, 1993).

Adriana Marshall (1988) señala que entre 1976 y 1983, el impacto de la política económica sobre la situación de los trabajadores fue regresivo, tendencia reforzada por las políticas sociales y la seguridad social que apuntaron a disminuir los costos laborales indirectos, a la vez que cambió la regulación de los salarios y las condiciones de contratación. Estos cambios inauguraron un nuevo escenario en las relaciones sociales entre sindicatos, Estado y empleadores, siendo la relación asalariada (que definimos anteriormente como el soporte social) la que se convierte en el eje de las transformaciones.

Con el modelo instalado en 1976 se inicia la decadencia de la protección del trabajo y el disciplinamiento de la fuerza laboral en un contexto donde el consumo del mercado interno deja de jugar un rol importante. En efecto, se desplaza la intervención estatal desde la protección del trabajo hacia una serie de medidas que pretendían fortalecer la prerrogativa empresarial. En este marco se propone reinstaurar la competencia en el mercado de trabajo y focalizar la intervención social del Estado en los indigentes, es decir, restringir la asistencia a los más pobres, dejando en los asalariados la responsabilidad del financiamiento y provisión de su propia seguridad social. Así, se iniciaron algunos procesos de descentralización en educación y salud.

El paradigma ideológico que se generalizó a partir de la década del 90 definía que la política social debía ajustarse al modelo de liberalización económica aplicado a la región (Cortés y Marshall, 1999). De este modo, distintos organismos internacionales presionaron para que América Latina inicie una drástica reestructuración de la política 
social. El paquete de reformas propuestas para Argentina, Chile y el Centro y Este Europeo, explica Esping-Andersen (1996), siguieron -en líneas generales- una estrategia liberal basada en la privatización de los sistemas jubilatorios, reducción de la red de seguridad social de origen público, el pasaje a programas asistenciales focalizados en función de recursos económicos individuales y la flexibilización del mercado laboral. Esas líneas generales fueron concordantes con el papel asignado al Estado: un Estado mínimo que no intervenga en el libre juego de los agentes económicos.

Se diluyó la responsabilidad social y la cobertura universal, prefiriendo una mayor selección y fragmentación de los programas que son diseñados en función a las especificidades de los grupos vulnerables o de alto riesgo, en donde no existe un "derecho a" sino un "programa para". Y la consecuencia de ello es la implementación de políticas focalizadas para los llamados grupos vulnerables en desmedro del fortalecimiento de las instituciones de política social de corte universalista y de acceso incondicional para todos los ciudadanos/as (Pautassi, 2000:126).

En términos teóricos, la focalización aparece como el correlato de la individualización de la fuerza de trabajo y de la posibilidad estructural de la exclusión de una parte de ella del mercado laboral. La política social deviene compensatoria de las inequidades desencadenadas por los efectos resultantes de la implementación, las políticas de ajuste macroeconómico y reforma estructural (Grassi, 1994).

En el caso argentino, todas estas transformaciones traen aparejado un desfasaje entre las demandas y las políticas sociales, que hasta el momento estaban dirigidas a canalizar otros tipos de conflictos. El resultado es un fuerte desequilibrio entre el riesgo social creciente y las políticas sociales, cada vez más restringidas. En efecto, los programas focalizados ofrecidos por el gobierno estaban diseñados para satisfacer las necesidades y demandas típicas de los pobres estructurales, dejando fuera a las clases medias empobrecidas.

La asociación entre desigualdad en la distribución del ingreso e inclusión social ha estado medida históricamente por el funcionamiento de las instituciones sociales, económicas y políticas, que han 
favorecido o coartado las oportunidades de satisfacción de necesidades y -sobre todo- de la práctica de ciudadanía. Como ya se dijo, en el caso argentino, la seguridad social estuvo profundamente ligada a la condición de trabajador, lo que ha derivado en una inclusión diferenciada en el sistema social. Esta forma de inclusión plantea una segmentación en lo que hace a la inserción de la población en el sistema social, que no ha revestido un carácter universal. A partir del advenimiento del neoliberalismo, esta segmentación emerge con mayor crudeza ante el progresivo desmantelamiento y mercantilización de los servicios sociales. La descentralización de servicios fundamentales, como la educación y la salud, ha derivado no sólo en una mayor inequidad, sino en una dramática profundización de las distancias sociales en función tanto del acceso a oportunidades (ya sea de empleo, de educación o de salud) como de la calidad de las oportunidades a las que se accede.

Dado que los diferentes ámbitos del sistema de protección social debían ser ocupados por el mercado (salud, educación, previsión, etc.) queda reservado para el Estado la política social no rentable, por supuesto, entre ellas, la asistencia, y el control social.

\section{La recomposición del sistema de protección}

A partir de 2003 el nuevo gobierno muda la estrategia en materia de políticas sociales. El crecimiento económico, la recuperación del empleo y la restitución de las instituciones del servicio social serán las principales herramientas de la estrategia de inclusión y movilidad social. De hecho se escucha repetir el slogan "La mejor política social es el trabajo" (Kirchner, 2010).

La ecuación se explicaba en las expectativas que los indicadores levantaban, ya que para el 2007 los índices que mostraban una alta recuperación del empleo, de hecho la desocupación había descendido a un dígito y se observaba un alto porcentaje de empleo registrado entre los nuevos puestos (Novick y Villafañe, 2008). 
Conscientes de la centralidad que aún tenía el trabajo en la vida social del país, el gobierno apuesta a la reconstrucción de ese, imperfecto, sistema de protección social argentino basado en el seguro social contributivo. En este marco se deroga la llamada Ley Banelco $\left(\mathrm{N}^{\circ} 25250 / 00\right)$ y se sanciona la nueva ley de empleo.

En relación a la política de empleo, una de las principales acciones del gobierno nacional fue sancionar en el 2004 el decreto $\mathrm{N}^{\circ} 1506$. El mismo establece que se prorrogue la Emergencia Ocupacional Nacional y el Plan Jefes y Jefas de Hogar. A su vez, pone un plazo de 180 días para que los Ministerios de Trabajo, Empleo y Seguridad Social (en adelante MTEySS) y del Ministerio de Desarrollo Social (en adelante MDS) realicen una clasificación de los beneficiarios del Programa Jefes de Hogar de acuerdo a las condiciones de empleabilidad.

En este sentido: en la órbita del MDS las personas incorporadas a partir de esta reglamentación se incorporaron al Plan "Familias por la Inclusión Social” y los considerados empleables se incorporaron a través del Ministerio de Trabajo al Seguro de capacitación y empleo. Sin entrar en el análisis de los planes en particular, los dos planes suponen un subsidio a los beneficiarios el primero en carácter de asistencia ante la situación de vulnerabilidad social y el otro en carácter de seguro por la situación de desempleo hasta tanto pueda incorporarse en el mercado de trabajo. Por lo que en términos de diseño de política los dos planes se caracterizan por ser subsidios a la demanda.

En consecuencia, para este entonces el MDS establecía como sus principales ejes de intervención social: "la familia", "el trabajo" y "la organización social". Que sea abordaban a través de: el plan "Familias por la inclusión social", el plan de "Seguridad Alimentaria" y el plan de "Desarrollo Social y Economía Social". Y más orientadas al tercer eje de intervención se desplegó una serie de estrategias de intervención comunitaria, de "cohorte desarrollista". Específicamente los Centros integradores comunitarios, los Centros de Referencia y el plan "Ahí" de abordaje integral ${ }^{3}$. Estos respondían, por un lado, a tratar de re-

3 Para mayor detalle sobre los diferentes planes ver: Alicia Kirchner. La Bisagra. Disponible en http:// 
articular del tejido social y fortalecer las identidades colectivas; y, por otro, a capitalizar lo que había sido el sustento político inicial del gobierno, que se asentaba en parte en el trabajo territorial de las organizaciones sociales de base. Estas instituciones territoriales permitían la ejecución de planes y programas de forma directa en territorio sin la intermediación de las provincias.

De manera similar, el MTEySS fortaleció y creó nuevas Oficinas y Unidades de Empleo. Y la ANSES desarrolló la estrategia territorial a través de sus diferentes delegaciones (UDAI - Unidad de Atención Integra, ULAT - Unidad Local Transitoria, ULADE - Unidad Local de Atención de Empresas, UDAM - Unidad de Atención Móviles, Terminales de Autoconsulta).

En el ámbito de la salud no se produjeron cambios significativos. En sintonía con la estrategia anterior el Ministerio de Salud desplego el Plan Nacer, que incluye equipamiento para el primer sector y el programa Médicos Comunitarios, que supone la inclusión de profesionales en el primer nivel de inserción, a través de una formación en servicio. Pero de entre las políticas de este Ministerio se destacan principalmente: la Ley de medicamentos genéricos, el programa Remediar y la intervención en salud sexual y procreación responsable. No obstante el gran problema que afecta al sistema de salud es su fragmentación entre sus subsectores público, privado y sindical, y entre las diferentes jurisdicciones. En este sentido se avanzó con revitalización del Concejo Federal de Salud, pero lejos se está de hacer mella en los enquistes institucionales y económicos del campo de la salud argentina que impacten en la morfología del sistema de protección.

En el ámbito de la educación se produjeron algunos cambios importantes, la Ley N 26075 de financiamiento educativo en 2005 impulsó la inversión en educación hasta poco más del 6\% del PBI en 2010. En 2006 se sancionó la Ley de Educación Nacional, en la que

www.desarrollosocial.gob.ar/Uploads/i1/Institucional/La_Bisagra_Politicas_Sociales_en_accion. pdf o Políticas Sociales del Bicentenario Tomos I y II. Disponible en: http://www.desarrollosocial. gob.ar/Uploads/i1/Familia Argentina/Pol\%C3\%ADticas\%20Sociales\%20del\%20Bicentenario\%20 -\%20Tomo\%20I.pdf y en: http://www.desarrollosocial.gob.ar/Uploads/i1/FamiliaArgentina/ Pol\%C3\%ADticas\%20Sociales\%20del\%20Bicentenario\%20-\%20Tomo\%20II.pdf 
se destaca la obligatoriedad de la educación secundaria así como la universalización de la sala de 4 años (Repetto y Chudnovsky, 2009). De todos modos, de las iniciativas del Ministerio de Educación, se destaca todo lo realizado en materia de comunicación e incorporación de nuevas tecnologías en el aprendizaje (Canal Encuentro y Pakapaka, Conectar igualdad, educ.ar, etc.). Sin embargo, queda un gran problema a resolver que tiene que ver con las condiciones de trabajo de los docentes. O sea, intervenir principalmente sobre las disparidades regionales, los salarios y la dispersión horaria.

En el ámbito de la previsión social, la sanción de la Ley $\mathrm{N}^{0} 25.994$ (conocida como Plan de Inclusión Previsional) abrió un nuevo escenario. La norma posibilitó la obtención de beneficios previsionales a las personas que acreditaran los años de servicio (30 años) sin tener la edad jubilatoria, o bien de aquellos que, acreditando la edad jubilatoria, no contaban con los años de servicio. En ese último caso, la implementación de una generosa moratoria facilitó el acceso de los potenciales beneficiarios (Rubinzal, 2008). De esta manera se da la primera ruptura con el principio contributivo del sistema de protección social.

A partir de la moratoria previsional se inaugura una nueva generación de políticas sociales del gobierno kirchnerista, que en parte iría a contramano de la inicial propensión a la territorialización y las políticas relacionadas al trabajo y al seguro social bismarkiano. La cual había encontrado límites en los, todavía, altos índices de trabajo informal, y en los núcleos persistentes de pobreza y exclusión. En este mismo sentido, a partir del 2004, desde el MDS se produjo un aumento sostenido de las pensiones no contributivas. Por lo menos hasta el año 2012 (últimos datos disponibles) donde llegaron a 1.276.949, aproximadamente, un $360 \%$ más en relación a las 344.630 de $2003^{4}$.

La moratoria previsional tuvo un alto impacto en el primero y segundo quintil de población, y particularmente, en las mujeres que no tenían todos sus aportes, de hecho se la conoció como "la jubi-

4 Ministerio de Trabajo y Seguridad Social: “Boletín estadístico de la Seguridad social”. Segundo trimestre 2012 . 
lación de amas de casa” (Danani \& Hinze, 2013). “En el año 2005 había 3.190.000 jubilados, a junio de 20105.600 .000 jubilados; hay 2.400.000 jubilados que se han incluido en el plan de inclusión jubilatoria, con una clara política de inclusión” (Bossio, 2010).

A este hecho se le sumaron los aumentos de las jubilaciones mínimas y en 2008 la Ley de Movilidad Jubilatoria, como antecedentes de la reestatización de los fondos jubilatorios a fines del mismo año, lo que significó un profundo cambio en lo que a políticas sociales respecta. En principio, implicaba dar vuelta atrás con la reforma neoliberal, recuperar el dinero de los trabajadores del sistema financiero y sobre todo recuperar el principio solidario del sistema.

Pero también significo dotar al Estado de recursos para recuperar la gobernabilidad sobre instrumentos de política económica y social. Y así lo entendió el gobierno que a partir de esta medida inauguró la nueva generación de políticas sociales, con el decreto de creación de la reclamada Asignación Universal por Hijo (en adelante AUH).

Al momento del lanzamiento de la AUH, la estrategia desarrollada había permitido recuperar los mismos niveles de bienestar social observados al comienzo del Plan de Convertibilidad. Pese a ello, tanto los indicadores laborales y distributivos, como la incidencia de la pobreza y la indigencia se encontraban aún demasiado alejados de los niveles deseables (Agis et al, 2010).

Por tales motivos, hacia fines de 2009 el gobierno nacional pone en marcha dos programas concomitantes que intentan recuperar los estándares sociales de la década del setenta: la Asignación Universal por Hijo para Protección social (AUH del ANSES) y el Programa Ingreso Social con Trabajo, Argentina Trabaja (del MDS) (Agis et al, 2010).

El Plan Argentina Trabaja no supuso, más allá de su envergadura y la inmensidad de críticas y opiniones que suscitó, un cambio radical en relación a otras políticas de empleo. Concretamente, este programa se propone la creación de cooperativas de trabajo, integradas por al menos 60 personas, a fin de que las mismas realicen obras públicas vinculadas con labores de baja y mediana complejidad para el mejoramiento de la infraestructura y el hábitat barrial de las localidades 
más vulnerables del país. Asimismo, el programa prevé la capacitación en oficios, en organización cooperativa y en temáticas sociales, educativas y de salud y prevención para los cooperativistas. Para ello se brinda asistencia técnica y becas de capacitación (Gimenez y Hopp, 2012).

Lo novedoso es la utilización de la figura de cooperativas para organizar los grupos de trabajo. Lo cual fue muy criticado desde el movimiento cooperativista, por no respetar los principios del cooperativismo, y más ampliamente por utilizar la figura de asociados, en tanto cooperativistas y no de trabajadores, lo que supone una restricción de derechos a partir de una relación laboral inexistente o lábil ${ }^{5}$.

No es objeto de este trabajo abordar el análisis de los planes y programas en concreto, sino que, más allá de las particularidades, interesa destacar que este programa se lanzó en conjunto con la AUH, el cual sí talla profundo en el principio contributivo del sistema de protección social argentino. La extensión a los trabajadores informales y desocupados de la AUH implicó la universalización de las asignaciones familiares a todos los niños más allá de si sus padres trabajan o no; o si hacen o no aportes al sistema.

La AUH no fue pensada como un plan, sino como parte integrante de la seguridad social, aunque al ser incompatible con cualquier otro plan, estos fueron absorbidos y sustituidos. Sin embargo, la asignación se financia básicamente con recursos provenientes del ANSES.

En cuanto al impacto, el estudio de Danani \& Hintze (2013) señala que existe entre un 13 y un $18 \%$ de la niñez y adolescencia urbana que no satisface sus "necesidades básicas". Es hacia ese segmento etario donde se dirige la AUH. Esa estimación incluye más de tres millones de AUH, lo que representa más del 30\% del segmento de hasta 18 años.

Ya desde su concepción, con cumplimiento satisfactorio en la implementación, la AUH tiene un sesgo progresivo en lo que hace a los destinatarios: llega, sin duda, a los quintiles de menores ingresos. Pero también señalan los límites de la cobertura ya que entre 500.000

5 Para profundizar sobre el tema ver Vuotto (2011). 
y un millón de Niños, niñas y adolescentes, no son alcanzados por la asignación, y estimaciones del Barómetro de la Deuda Social indicaban en 2011 que, cerca de un 9\% de ellos y ellas estaban en situación de pobreza extrema ${ }^{6}$.

A su vez, según los datos disponibles, la AUH ha mantenido su cobertura en alrededor de los 3.500 .000 beneficiarios con una leve tendencia hacia la baja desde 2011 (3.531.181 en Junio de 2010, 3.595.163 en Junio de 2011 y 3.424.458 Junio de 2012). Sin embargo, las asignaciones familiares por hijos del sector activo han tenido un importante y sostenido descenso del $22 \%$ de beneficiarios (3.283.253 en Julio de 2010, 3.053.241 Junio de 2011 y 2.557.330 en Junio de 2012). Ello probablemente tenga que ver con la desactualización de los montos máximos para el cobro de la asignación dado que los aportantes al sistema previsional vienen aumentando sostenidamente lo que supone un aumento del trabajo registrado (8.636.122 en Diciembre de 2009, 9.023.893 en Diciembre de 2010, 9.397.305 en Diciembre de 2011 y 10.553 .566 en Junio de 2012) ${ }^{7}$.

Además de la AUH, luego de la reestatización de los fondos previsionales, se crearon otros tres grandes programas nuevos: $\mathrm{El}$ Programa Crédito Argentino del Bicentenario para la vivienda única familiar (Pro.Cre.Ar) con el objetivo de "facilitar el acceso a la vivienda propia de la población y la generación de empleo como políticas de desarrollo económico y social" ${ }^{8}$. Se crea un fondo fiduciario con aportes del Ministerio de Economía el ANSES y privados, a partir de la creación de un bono de deuda pública para brindar 400.000 créditos con el fin de financiar la construcción de vivienda. Obviamente tiene a su vez el objetivo de impulsar la actividad económica y crear empleo.

El "Programa de Respaldo a Estudiantes Argentinos" (PROGRESAR), con el fin de generar nuevas oportunidades de in-

6 Danani \& Hintze (2013).

7 Ministerio de Trabajo y Seguridad Social: "Boletín estadístico de la Seguridad social”. Segundo trimestre 2012.

8 Decreto 902 de 2012. Boletín Oficial de la República Argentina, miércoles 13 de junio de 2012. Disponible en http://procrear.anses.gob.ar/documentos/decreto-procrear.pdf 
clusión social y laboral a los jóvenes en situación de vulnerabilidad, a través de acciones integradas que permitan su capacitación e inserción laboral ${ }^{9}$. Este es un programa para jóvenes desocupados ${ }^{10}$ de entre 18 y 24 años que consiste en la transferencia de una suma de 600 pesos (mensuales) para los que decidan iniciar o retomar los estudios.

En el 2013 el MDS complementa el Programa de Ingreso Social con Trabajo "Argentina Trabaja" con una línea específica orientada a las mujeres desocupadas, priorizando jefas de hogar con hijos discapacitados o tres o más hijos a cargo, o que padecen violencia de género, y viven en villas y asentamientos o barrios emergentes; por considerar que constituyen el "núcleo duro" de la vulnerabilidad y exclusión socio-ocupacional. La propuesta es a través de este programa incluir a 100.000 mujeres de todo el país puedan acceder a un trabajo mediante de la conformación de cooperativas, y a la posibilidad de capacitarse y culminar sus estudios. Primero se comenzó por las localidades del plan "Ahí" y luego se continuó ampliando a otros distritos $^{11}{ }^{12}$. El general el programa ingreso social con trabajo (incluido "Ellas Hacen”) se gestiona a través de las Provincias y los Municipios son quienes definen las obras a realizar y organizan la inscripción de los interesados. En varios casos, los municipios abren la participación a organizaciones sociales para formar sus cooperativas y definir obras en los barrios en los que trabajan.

9 Decreto $\mathrm{N}^{\circ} 84$ de 2014.

10 Según el decreto, el Programa está destinado específicamente a los jóvenes de entre dieciocho (18) y veinticuatro (24) años de edad inclusive residentes en la República Argentina, siempre que los mismos o sus grupos familiares se encuentren desocupados o se desempeñen en la economía informal o formal, o sean titulares de una prestación provisional contributiva o pensión no contributiva o monotributistas sociales o trabajadores de temporada con reserva de puesto o trabajadores del Régimen de Casas Particulares.

11 García, E. "Programa Ellas hacen: trabajo asociado y terminalidad educativa". Observatorio Social de Empresas Recuperadas y Autogestionadas. Disponible en http://webiigg.sociales.uba.ar/ empresasrecuperadas/PDF/PDF_09/Informe_Coyuntura_Ellas_Hacen.pdf

12 Ministerio de Desarrollo Social: " $1^{\circ}$ Informe Antecedentes, creación y primera etapa de Ellas Hacen”, Abril 2014. Disponible en: http://www.desarrollosocial.gob.ar/Uploads/i1/1er\%20 informe, $\% 20$ antecedentes $\% 20$ creacion $\% 20 y \% 201$ er $\% 20$ etapa.pdf 


\section{Conclusiones}

El sistema de protección social argentino actual se puede caracterizar como mixto. Es decir, en la batería actual de políticas sociales, se observa una referencia a los diferentes tipos de políticas sociales más o menos articuladas.

A partir de la reactivación económica, en los años posteriores a la crisis del 2001, se apostó a la reconstrucción de sistema de protección social basado en el seguro social contributivo. A la vez que se llevó adelante un despliegue territorial, que respondió, en parte, a la estrategia de saltar las jurisdicciones (provincias y municipios) y tener relación directa con actores sociales locales para abordar problemáticas y articular la asistencia en el territorio.

En el ámbito de la seguridad social, se han dado las acciones más importantes del período en términos de política social, con la recuperación de los fondos de jubilaciones y pensiones se produce la recomposición del sistema de protección social que incluye un enfoque más inclusivo, el cual se evidencia en la gran cobertura y la magnitud de las transferencias monetarias concretamente transferidas. Específicamente la AUH, la moratoria previsional y las Pensiones No Contributivas. La AUH es un salto cualitativo en relación a la responsabilidad del Estado en abordar el problema de la pobreza de lleno, intentado garantizar un mínimo de subsistencia por derecho de ciudadanía, que revierta una desigualdad histórica entre trabajadores ocupados y desocupados.

Con la recuperación de los fondos provisionales, la ANSES llevó adelante la AUH y pasó a ser el organismo protagónico en la intervención social del Estado. Desplegando una batería de programas de gran envergadura como PRO.CRE.AR Y PROGRESAR.

No obstante, la pobreza excede el problema de los ingresos e incluye una infinidad de problemas sociales asociados que, de una forma u otra, lesionan la integración social. Esta situación exige pensar un sistema de protección social, integrado e integral, que pueda articular respuesta a la diversidad de sujetos y de situaciones problemáticas. 


\section{Referencias bibliográficas}

AGIS, E, CAÑETE, C., PANIGO, D. "El impacto de la Asignación Universal por Hijo en la Argentina", 2010. [En línea: 15/05/2014] Disponible en: http://www.trabajo.gov. ar/left/estadisticas/DocumentosSUBWEB/area1/documentos/AUH_en_Argentina.pdf, 24/11/12.

AMBITO.COM. "Bossio destacó que este año se invertirá más de 10\% del PBI en seguridad social”, 15 de Junio de 2010. [En línea: 16/04/2014] Disponible en: http:// www.ambito.com/noticia.asp?id=527668\&seccion=Econom\%EDa\&fecha=15/06/2010. Consultado: marzo de 2014.

ARIAS, A. Pobreza y modelos de intervención. Aportes para la superación del modelo de asistencia y promoción. Buenos Aires, Espacio, 2012.

BUSTELO, E. y MINUJIN, A. Todos entran. Buenos Aires, Planeta, 1998.

CELS. "Derechos humanos en Argentina”. Informe 2008. Buenos Aires, Siglo Veintiuno Editores, 2008.

CORTES, R y MARSHALL, A. "Estrategia económica, instituciones y negociación política en la reforma social de los noventa", en: Desarrollo Económico. Vol. 39, N 154, Buenos Aires, julio-septiembre 1999.

DANANI, C. y HINTZE, S. "Seguridad social y condiciones de vida. La protección social en la Argentina entre 2002 y 2012”, en: Revista Voces en el Fénix, Año 4, ํ2 23, 2013.

DRAIBE, S. y RIESCO, M. Estado de bienestar, desarrollo económico y ciudadanía: algunas lecciones de la literatura contemporánea. Serie "estudios y perspectivas", CEPAL Unidad de Desarrollo Social. México, 2006.

ESPING-ANDERSEN, G. Fundamentos sociales de las economías postindustriales. Barcelona, Ariel, 2002.

FLEURY, S. y MOLINA, C. G. "Modelos de Protección Social”. Notas de clase. Washington, D.C. Banco Interamericano de Desarrollo, Instituto Interamericano para el Desarrollo Social (INDES), 2000. [En línea: 04/03/2013] Disponible en: http://indes. adb.org/pub.asp

FONSECA, A. Los sistemas de protección social en América Latina. Un análisis de las transferencias monetarias condicionadas. RBLAC, PNUD, 2006. [En línea: 21/03/2013] Disponible en: http://files.uladech.edu.pe/Virtual_Educa/DARES_PL_2010/sesion5] protecci $\%$ C3\%B3n $\% 20$ social $\% 20$ en $\% 20$ el $\% 20$ per $\%$ C3\%BA.pdf

GARCÉS, L. y LUCERO, M. "Nuevos desafíos para el Estado y la ciudadanía: La recomposición del campo de la asistencia en Argentina”. Congreso de Cátedras de Estructura Social, Problemas Sociales en Carreras de Trabajo Social. Facultad de Trabajo Social, La Plata, 9 y 10 de Octubre de 2008. 
GOLBERT, L; ROCA, E. E; LANARI, M. E. ¿Piso o Sistema de Integrado de Protección Social? Una Mirada desde la Experiencia Argentina. $1^{\circ} \mathrm{ed}$. Buenos Aires, Ministerio de Trabajo y Seguridad Social. Secretaría de Seguridad Social, 2012. ISBN 978-987-25026-7-6

GRASSI, E.; HINTZE, S.; NEUFELD, M. Políticas Sociales, Crisis y Ajuste Estructural. Buenos Aires, Espacio Editorial, 1994.

ISUANI, E. “Bismarck o Keynes: ¿Quién es el Culpable?”, en: Isuani, E. y otros. El Estado de Bienestar: la crisis de un paradigma. Buenos Aires, CIEPP, Miño Dávila Editores, 1991.

KIRCHNER, A. Políticas sociales del Bicentenario. Un modelo nacional y popular. Tomo I. Buenos Aires, Ministerio de Desarrollo Social de la Nación, 2010.

MESA-LAGO, C. Desarrollo de la Seguridad Social en América Latina. CEPAL, 1985. [En línea:01/02/2014] Disponible en: http://es.scribd.com/doc/122667408/Mesa Lago-Carmelo-El-Desarrollo-de-La-Seguridad-Social-en-America-Latina-Santiago-deChile-1985.

MARSHALL, A. Políticas sociales: el modelo neoliberal. Argentina (1976-1983). Buenos Aires, FLACSO/LEGASA, 1988.

NOVICK, M. y VILLAFAÑE, S. "El trabajo como factor constitutivo de la ciudadanía", en: NOVICK, M. PEREZ SOSTO G. (coord.). El Estado y la reconfiguración de la protección social. Buenos Aires, Siglo XXI Editores, ITDT-Cátedra UNESCO-MTESS, 2008.

OSLACK, O. "El mito del Estado mínimo: una década de reforma estatal en Argentina". Trabajo presentado al V Congreso Internacional del CLAD sobre la Reforma del Estado y de la Administración Pública, Santo Domingo, Rep. Dominicana, 24-27 Oct. 2000. [En línea: 27/05/2007] Disponible en: www.unpan.org

PAUTASSI, L. "El impacto de las reformas estructurales y la nueva legislación laboral sobre la mujer en Argentina", en: BIRGIN, H. (comp.) El derecho en el género y el género en el derecho. Buenos Aires, CEADEL, 2000.

REPETTO, F. y CHUDNOVSKY, M. "Las políticas sociales en la Argentina reciente. Avances y desafíos desde una perspectiva progresista", en: QUIROGA, CANZANI y ENSIGNIA (Comp.). Consenso progresista: Las politicas sociales de los gobiernos progresistas del Cono Sur. Uruguay, Friedrich Ebert Stiftung, 2009.

RUBINZAL, D. "Abuelos bajo el paraguas. Aumenta la cobertura previsional" en: Suplemento "Cash", Diario Página/12, 2 de Marzo de 2008. [En línea: 03/03/2014] Disponible en: http://www.pagina12.com.ar/diario/suplementos/cash/17-3400-2008-03$02 . \mathrm{htm}$

SCHNEIDER, S. y PEYRÉ TARTARUGA, I.G. "Territorio y enfoque territorial: de las referencias cognitivas a los aportes aplicados al análisis de los procesos sociales rurales", en: MANZANAL, M.; NEIMAN, G. y LATTUADA, M. (Org.). Desarrollo Rural. Organizaciones, Instituciones y Territorio. Buenos Aires, Ed. Ciccus, 2006. Pp. 71-102. 
VUOTTO, M. "Acerca del cooperativismo de trabajo en la Argentina", en: Revista Voces en el Fénix, ¿La clase obrera va al Paraíso? Año 2, N 6, junio 2011. Facultad de Ciencias Económicas, UBA.

Recepción: 14/05/2015

Aceptación: 04/07/2015 